\title{
Promoter hypermethylation silences cyclooxygenase-2 (Cox-2) and regulates growth of human hepatocellular carcinoma cells
}

\author{
Hiroaki Murata ${ }^{1}$, Shingo Tsuji ${ }^{1}$, Masahiko Tsujii ${ }^{1}$, Yasuko Sakaguchi ${ }^{2}$, Hai Ying Fu ${ }^{2}$, \\ Sunao Kawano ${ }^{2}$ and Masatsugu Hori $^{1}$ \\ ${ }^{1}$ Department of Internal Medicine and Therapeutics and ${ }^{2}$ Department of Clinical Laboratory Science, School \\ of Allied Health Sciences, Osaka University Graduate School of Medicine, Osaka, Japan
}

\begin{abstract}
Cyclooxygenase-2 (COX-2) upregulation is recognized to confer advantage in progression in a wide variety of cancers, with colorectal cancer most intensively investigated. Epidemiologically, chemopreventive effects of COX-2 inhibitors have been proven on numerous cancers, but not on hepatocellular carcinoma (HCC). Although the antiapoptotic feature of COX-2 generally supports cancer cell growth, previous reports have shown that COX-2 expression, upregulated in early HCC, is downregulated in advanced HCC. Therefore, COX-2 downregulation may be somehow advantageous and specific for HCC development. However, its mechanism remains unclear. Since promoter hypermethylation often silences the gene expression, we hypothesized that the epigenetic mechanism might regulate COX-2 expression in HCC. We examined the methylation status of the Cox-2 promoter in six human HCC cell lines (Hep3B, HepG2, SK-Hep1, HuH7, PLC, and FLC-7 cells) using methylation-specific PCR. The promoter was remarkably hypermethylated in Hep3B and FLC-7 cells and moderately in HepG2 and SK-Hep1 cells, but not in HuH7 and PLC cells. In Hep3B cells, coincubation with 5-aza2'-deoxycytidine, a demethylator, demethylated the promoter and upregulated COX-2 expression as well as prostaglandin $E_{2}$ production dose dependently. On the other hand, no such effects were observed in HuH7 cells. Additionally, the methylator suppressed growth of Hep3B cells dose dependently, accompanied by cyclin D1 downregulation, and the growth suppression was abrogated by potent COX-2 inhibition with a COX-2 selective inhibitor celecoxib, but these responses were not found in $\mathrm{HuH7}$ cells. These results indicated that cell growth was largely retarded by Cox-2 upregulation via promoter demethylation, rather than the potentially reactivated genes concurrently demethylated by 5 -aza-2'-deoxycytidine. In conclusion, promoter hypermethylation transcriptionally silences Cox-2 in HCC cells. Epigenetic alteration of Cox-2, at least in part, modulates the growth of HCC cells.
\end{abstract}

Laboratory Investigation (2004) 84, 1050-1059, advance online publication, 24 May 2004; doi:10.1038/labinvest.3700118

Keywords: COX-2; hypermethylation; HCC; cell growth; 5-aza-2'-deoxycytidine

Genetic changes, such as mutations and translocations, and epigenetic changes, without a change in DNA sequence, play a major role in carcinogenesis. DNA methylation is one of epigenetic changes and hypermethylation of $\mathrm{CpG}$ islands in the promoter region is a powerful mechanism for the suppression of gene activity by transcriptional silencing. ${ }^{1-3}$

Correspondence: Dr Sunao Kawano, MD, PhD, Department of Clinical Laboratory Science, School of Allied Health Sciences, Osaka University Graduate School, 1-7 Yamada-Oka, Suita-City, Osaka 565-0871, Japan.

E-mail: kawano@sahs.med.osaka-u.ac.jp

Received 6 October 2003; revised and accepted 23 March 2004; published online 24 May 2004
In normal cells, methylation of $\mathrm{CpG}$ islands generally occurs only in two circumstances: on the inactive $\mathrm{X}$ chromosome in female somatic cells and on silenced alleles of parentally imprinted genes. ${ }^{4,5}$ Alterations of global DNA methylation have been found more often in a variety of cancers than in normal postnatal tissues, ${ }^{1,6,7}$ and aberrant DNA methylation is implicated in tumorigenesis. ${ }^{8,9}$ Especially, aberrant hypermethylation of the promoter of tumor suppressor genes can contribute to the formation and progression of cancer. ${ }^{1,2}$ This inappropriate gene silencing affects virtually every step in tumor progression. ${ }^{3}$

Cyclooxygenase (COX) is a rate-limiting enzyme in the production of prostaglandins (PGs) and thromboxanes, and COX-2 is inducible by a variety 
of cytokines, hormones, and tumor promoters. ${ }^{10-13}$ Upregulation of COX-2 has been demonstrated in various tumor tissues such as colorectal, ${ }^{14}$ gastric, ${ }^{15}$ esophageal, ${ }^{16}$ pancreatic, ${ }^{17}$ and lung cancers. ${ }^{18}$ COX-2 overexpression harbors antiapoptotic ${ }^{19}$ and pro-angiogenic ${ }^{20}$ properties as well as high metastatic potentials. ${ }^{21,22}$ COX-2 upregulation has also been reported in hepatocellular carcinoma (HCC) tissue and cell lines ${ }^{23-28}$ and in non-tumorous liver tissue. ${ }^{24,28,29}$

Interestingly, early HCC expresses COX-2 more frequently and strongly than advanced HCC. ${ }^{24,26-28}$ Meanwhile, we have previously reported that COX2 is overexpressed in advanced gastric cancer rather than early cancer. ${ }^{15}$ Therefore, the generally accepted idea that COX-2 is overexpressed along cancer development is not directly applied to HCC, and COX-2 overexpression may play a major role in the oncogenic process in HCC but have a different function in advanced HCC.

COX-2 upregulation allows advantages for tumor cells to grow, but at the same time causes cell cycle arrest in a variety of cells. ${ }^{30,31}$ In other words, COX-2 upregulation may suppress proliferation in the short term, but ultimately supports tumor growth in the long term. $\mathrm{PGE}_{2}$ is a major metabolite of arachidonic acid and its production is catalyzed by COX-2. E series PGs inhibit cell growth in melanoma, ${ }^{32}$ neuroblastoma, ${ }^{33}$ gastric cancer, ${ }^{34,35}$ and colonic epithelial cells. ${ }^{36,37}$ In contrast, it has also been reported that $\mathrm{PGE}_{2}$ increases cell motility and alters colony morphology, ${ }^{38}$ and has growth-promoting activity via transactivation of EGF receptor. ${ }^{39}$ Therefore, COX-2 upregulation and increased $\mathrm{PGE}_{2}$ production exert diverse actions in carcinogenesis, and clarification of the regulation of COX-2 expression is inevitable to better understand the mechanism of cancer development. Given that COX-2 expression changes over HCC progression, it is undoubtedly important to elucidate the mechanism of COX-2 regulation.

COX-2 inhibitors or nonsteroidal antiinflammatory drugs (NSAIDs) are known to reduce clinically the risk of fatal cancer such as esophageal, gastric, and colorectal cancer. ${ }^{40-42}$ Unfortunately, epidemiological evidence is lacking as to the growth-inhibitory effects of COX-2 inhibitors on HCC, and whether or not COX-2 inhibitors harbor chemopreventive properties on HCC has yet to be investigated.

Recently, Cox-2 promoter hypermethylation in colorectal and gastric cancer cell lines has been reported. ${ }^{43-45}$ In cases of HCC, a variety of genes are abnormally methylated, including Cox-2 promoter. ${ }^{46}$ However, it has yet to be investigated whether hypermethylation of the Cox-2 promoter indeed regulates its gene expression and modulates growth of HCC cells.

In this study, we explored the significance of the Cox-2 promoter hypermethylation in HCC cells using methylation-specific PCR (MSP). In addition, we investigated the effect of treatment with a demethylating agent 5-aza-2'-deoxycytidine (5-AzaCdR) on HCC cells. This study provided new evidence that hypermethylation of the Cox-2 promoter silenced the gene expression and the mechanism might contribute to the alteration of cell growth. To our knowledge, this is the first report to demonstrate the regulatory mechanism of Cox-2 by promoter hypermethylation and implicate its significance in the growth modulation of HCC cells.

\section{Materials and methods}

\section{Cell Culture}

Experiments were performed on six human HCC cell lines: Hep3B, HepG2, SK-Hep1, HuH7, PLC, and FLC-7 cells. HepG2 cells were established from a human hepatoblastoma, and the remaining five cell lines were derived from human HCC arising in adults. HepG2, SK-Hep1, and HuH7 cells do not have detectable hepatitis $\mathrm{B}$ viral sequences, while Hep3B and PLC cells have integrated HBV sequences. ${ }^{47}$ Hep3B cells have a partial deletion of the p53 gene while HuH7 cells have a point mutation in p53. ${ }^{48}$ They were obtained from the American Type Culture Collection, and grown in DMEM (Sigma Chemical Co., St Louis, MO, USA) supplemented with $10 \%$ fetal bovine serum (FBS) (Sigma) and $0.5 \%$ Antibiotics-Antimycotic ${ }^{\mathbb{R}}$ (Invitrogen Corp., Carlsbad, CA, USA) at $37^{\circ} \mathrm{C}$ in $5 \% \mathrm{CO}_{2}$ atmosphere.

\section{Methylation-Specific PCR}

DNA methylation patterns in the Cox-2 promoter were determined by MSP as essentially described by Kane et $a .^{49}$ Briefly, cells were cultured to subconfluence and harvested to extract genomic DNA using the QIAamp DNA blood mini kit (Qiagen, Germany). DNA $(1 \mu \mathrm{g})$ was incubated at $37^{\circ} \mathrm{C}$ for $24 \mathrm{~h}$ in a $50-\mu \mathrm{l}$ reaction for digestion containing $20 \mathrm{U}$ of $M s p \mathrm{I}, 20 \mathrm{U}$ of HpaII (New England Biolabs Inc., Beverly, MA, USA), or no enzyme. PCR was carried out in a $25-\mu$ l reaction mixture containing $2 \mu \mathrm{l}$ of each digest and $1 \mathrm{U}$ of AmpliTaq Gold DNA polymerase (Applied Biosystems, Foster City, CA, USA). Three sets of primers were designed to amplify the Cox-2 promoter sequence containing one $\mathrm{CpG}$ site each: -431 nucleotides (nt) (A), $-372 \mathrm{nt}(\mathrm{B})$, and $-138 \mathrm{nt}$ (C) from the transcription starting site to produce 159 , 101, and $111 \mathrm{bp}$ amplicons, respectively. Primer sequences were: (A) 5'-TTCTTAACCTTACTCGCCCCAG-3' (sense) and 5'-AGAAGGACACTTGGCTTCC TC-3' (antisense); (B) 5'-AGGAAGCCAAGTGTCCT TCT- $3^{\prime}$ (sense) and $5^{\prime}$-TTCTTCTTCGCAGTCTTTGC- ${ }^{\prime}$ (antisense); and (C) $5^{\prime}$-TAAAAAACCCTGCCCCCA-3 (sense) and 5'-GCCCATGTGACGAAATGACT-3' (antisense). After $10 \mathrm{~min}$ of initial heat denaturing at $95^{\circ} \mathrm{C}$, DNA was amplified by 30 cycles of denaturing at $95^{\circ} \mathrm{C}$ 
for $15 \mathrm{~s}$ and annealing and extension at $58^{\circ} \mathrm{C}$ for $60 \mathrm{~s}$, followed by $7 \mathrm{~min}$ at $72^{\circ} \mathrm{C}$. To ensure the reproducibility, the same experiments were performed three times.

\section{RT-PCR}

RNA was extracted from cultured cells using the RNeasy Mini kit (Qiagen). Reverse transcription was carried out with $1 \mu \mathrm{g}$ of total RNA as a template with ReverTra Ace- $\alpha$ - (Toyobo, Japan) according to the manufacturer's instructions. Then, cDNA was amplified by PCR with primers specific for Cox-2 and GAPDH as described by Takahashi et al. ${ }^{50}$ RT-PCR for $C O X-2$ and GPADH was carried out in a solution containing $1 \mu \mathrm{l}$ of cDNA, 1 x PCR buffer, $200 \mu \mathrm{M}$ of each deoxynucleotide triphosphate, $0.4 \mu \mathrm{M}$ of each primer, and $1 \mathrm{U}$ of Taq DNA polymerase (Qiagen).

\section{Western Blot Analysis}

After washing the cells with PBS $(9.57 \mathrm{mM}$, $\mathrm{pH}=7.35-7.65)$, HCC cells were harvested and lysed in RIPA buffer $(1 \times \mathrm{PBS} / 1 \%$ Nonidet P-40/ $0.5 \%$ sodium deoxycholate $/ 0.1 \%$ SDS) containing $0.1 \mathrm{mg} / \mathrm{ml}$ of PMSF and $51 \mu \mathrm{g} / \mathrm{ml}$ of aprotinin as final concentrations, followed by incubation on ice for $1 \mathrm{~h}$ and centrifugation at $4^{\circ} \mathrm{C}$ for $20 \mathrm{~min}$ at 12000 g. Protein concentrations of the cell lysates and supernatants were determined by the DC Protein Assay kit (Bio-Rad Laboratories, CA, USA). Then, $50 \mu \mathrm{g}$ of protein samples was resolved on $10 \%$ SDS-polyacrylamide gels and transferred to PVDF membranes. After blocking with 5\% nonfat powdered milk in TBS containing $0.1 \%$ Tween 20 (TBS$\mathrm{T}$ ) for $1 \mathrm{~h}$, the membrane was incubated overnight at $4^{\circ} \mathrm{C}$ with anti-human COX-2 antibody (Santa Cruz Biotechnology Inc., Santa Cruz, CA, USA), anticyclin D1 antibody (Santa Cruz), or GAPDH (Chemicon, Temecula, CA, USA). The membrane was washed with TBS-T three times and incubated with anti-rabbit immunoglobulin G (DAKO, Denmark) for $1 \mathrm{~h}$. After washing with TBS-T again, protein was visualized by the SuperSignal ${ }^{\circledR}$ chemiluminescence system (Pierce, Rockford, IL, USA).

\section{Treatment with 5-Aza-CdR and Celecoxib}

Cells were seeded at a density of $1 \times 10^{5}$ cells/ $100 \mathrm{~mm}$-dish and allowed to attach over $24 \mathrm{~h}$. To the medium, 5-Aza-CdR (Sigma) was added at a final concentration of 1,5 , and $10 \mu \mathrm{M}$, and cells were cultured for $96 \mathrm{~h}$. The medium was replaced every $24 \mathrm{~h}$ with a newly prepared medium containing 5-Aza-CdR.

To examine the effect of COX-2 inhibition, celecoxib (Pharmacia, St Louis, MO, USA), a COX2-selective inhibitor, was added at a final concentration of $1 \mu \mathrm{M}$ and cells were cultured for $96 \mathrm{~h}$ with different concentrations of 5-Aza-CdR. Then, the supernatant was collected for the measurement of $\mathrm{PGE}_{2}$.

\section{$\mathrm{PGE}_{2}$ Measurement}

After cells were treated with 5-Aza-CdR at different concentrations for $96 \mathrm{~h}$, the supernatant was collected for $\mathrm{PGE}_{2}$ measurement by an ELISA kit (Cayman Chemical, Ann Arbor, MI, USA). The concentrations of $\mathrm{PGE}_{2}$ were expressed as $\mathrm{pg} / \mu \mathrm{g}$ cell protein.

\section{Cell Number}

Cells were seeded at a density of $2 \times 10^{3} / 100 \mu \mathrm{l} /$ well on 96 -well plastic plates (about $20 \%$ confluence) and were cultured at $37^{\circ} \mathrm{C}$ in DMEM containing $10 \%$ FBS and different concentrations of 5-Aza-CdR. After incubation for $96 \mathrm{~h}$, WST-8 assay was carried out. After $10 \mu \mathrm{l}$ of the cell count reagent SF (Nacalai Tesque Inc., Japan) was added to each well and the cells were incubated for $3 \mathrm{~h}$ at $37^{\circ} \mathrm{C}$, the absorbance was measured at $450 \mathrm{~nm}$ with a reference wavelength at $630 \mathrm{~nm}$.

\section{Lactate Dehydrogenase Assay}

Lactate dehydrogenase (LDH) was measured to examine the cell viability after 5-Aza-CdR treatment. The culture medium was collected and centrifuged at $800 \mathrm{~g}$ for $10 \mathrm{~min}$ to obtain a cell-free supernatant. The supernatant was frozen for storage and sent to SRL Inc. (Tokyo, Japan) for measurement of LDH.

\section{Statistical Analysis}

The results were shown as mean \pm s.d. The statistical significance was determined by using Student's $t$-test. A $P$-value less than 0.05 was considered significant.

\section{Results}

\section{Cox-2 Promoter Hypermethylation in HCC Cell Lines}

The methylation status of the Cox-2 promoter was analyzed in six human HCC cell lines (Hep3B, HepG2, HuH7, PLC, FLC-7, and SK-Hep1). The Cox-2 promoter region contains 51 methylatable CpG sites spanning from -590 to $+186 \mathrm{nt}$ in relation to the transcription starting site $^{44}$ (Figure 1). This satisfies the established definition of a $\mathrm{CpG}$ island (776 bp fragments with a $\mathrm{G}+\mathrm{C}$ content $>0.57$ and an observed/expected presence of CpG >0.8). ${ }^{44,51,52}$ The methylation status was analyzed at three $\mathrm{CpG}$ sites, $-431 \mathrm{nt}(\mathrm{A}),-372 \mathrm{nt}(\mathrm{B})$, and $-138 \mathrm{nt}$ (C) by MSP. Genomic DNA was digested with a methylation-sensitive restriction enzyme HpaII, which could 


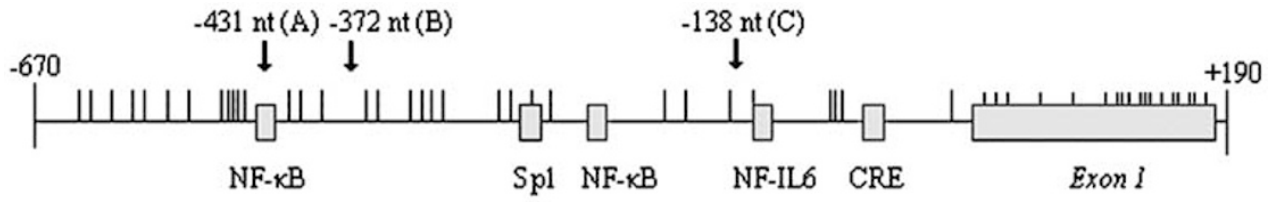

Figure $1 \mathrm{CpG}$ sites in Cox-2 promoter and targeted sites for MSP. The Cox-2 promoter contains 51 CpG sites. Three sets of primers were designed to amplify the Cox-2 promoter sequence containing one CpG site each: $-431 \mathrm{nt}$ (a), $-372 \mathrm{nt}$ (b), and $-138 \mathrm{nt}$ (c) from the transcription starting site. Transcription factor binding sites are also depicted.

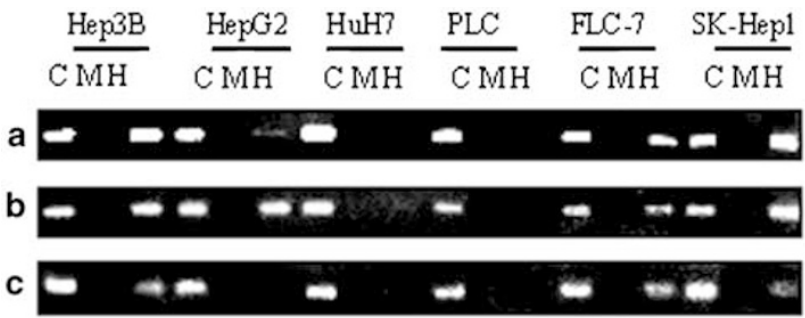

Figure 2 Cox-2 promoter hypermethylation in HCC cells. Hypermethylation of the Cox-2 promoter was examined by MSP in six HCC cell lines. At the three CpG sites of (a), (b), and (c), MSP produced 159, 101, and 111 bp amplicons, respectively. "C'”, "M", and "H" represent the amplification of the control (no restriction enzyme), MspI (methylation insensitive), and HpaII (methylation sensitive) digest, respectively. The presence of a band in the "H" column indicates that the CpG site is methylated. To confirm the cleavable $\mathrm{CpG}$ site without mutation, $M s p I$ digest was subject to MSP. The demonstrated figure was a representative result of three independent experiments that provided essentially similar data.

cleave a $\mathrm{CpG}$ site when it was not methylated. To ensure that the CpG site was not mutated and cleavable, the same DNA was digested with a methylation insensitive restriction enzyme MspI, which could cleave the CpG site irrespective of the methylation status. In short, when the CpG site was methylated, amplification of the HpaII digest should produce a band similar to the one in the control batch (without any restriction enzyme).

As shown in Figure 2, the methylation status at the three sites tended to be similar in each cell line. The promoter in HuH7 and PLC cells was unmethylated at these three sites. In contrast, the promoter in Hep3B and FLC-7 cells was remarkably hypermethylated, while that in HepG2 and SK-Hep1 cells was moderately hypermethylated. Some CpG sites such as (A) in HepG2, and (C) in Hep3B, FLC-7, and SKHep1 cells seemed to be half methylated.

\section{5-Aza-CdR Treatment Reactivated the Silenced COX-2}

Hep3B cells with the hypermethylated Cox-2 promoter and HuH7 cells with the unmethylated promoter were treated for $96 \mathrm{~h}$ with different concentrations of a demethylating agent 5-Aza-CdR. As shown in Figure 3a, although 5-Aza-CdR treatment at $1 \mu \mathrm{M}$ had little effect, the agent demethylated the promoter dramatically at 5 and $10 \mu \mathrm{M}$ at all the $\mathrm{CpG}$ sites examined in Hep3B cells.

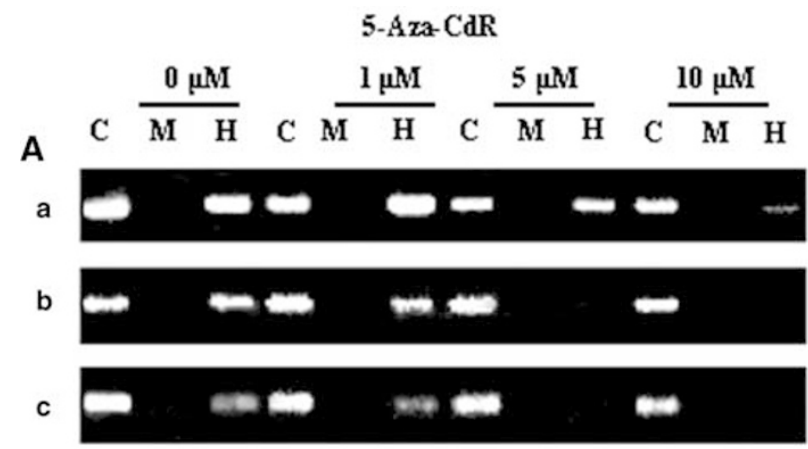

B

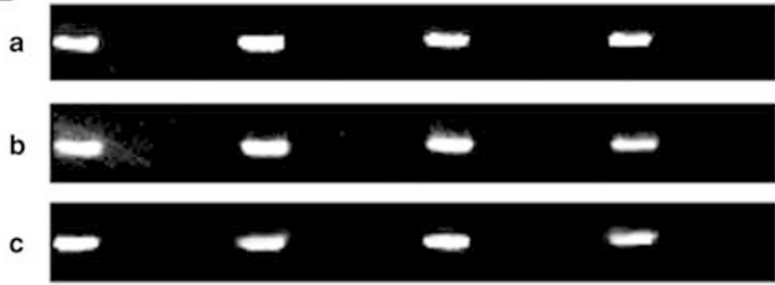

Figure 3 Demethylation by 5-Aza-CdR treatment. Hypermethylation in the Cox-2 promoter was examined by MSP after $96 \mathrm{~h}$ of treatment with 5-Aza-CdR at 1, 5, and $10 \mu \mathrm{M}$ in Hep3B cells (a) and $\mathrm{HuH} 7$ cells (b) At all the three $\mathrm{CpG}$ sites, 5-Aza-CdR significantly demethylated the promoter in Hep3B cells in a dose-dependent manner. No such change was observed in HuH7 cells. The experiments were repeated three times and essentially similar data were obtained. Representative results were shown.

On the other hand, as was expected, all the examined $\mathrm{CpG}$ sites remained unmethylated in HuH7 cells after 5-Aza-CdR treatment (Figure 3b). At a concentration of $20 \mu \mathrm{M}$, the agent was cytotoxic enough to detach both Hep3B and HuH7 cells from the plate (data not shown).

As shown in Figure 4a, 5-Aza-CdR treatment for $96 \mathrm{~h}$ stimulated COX-2 protein synthesis in Hep3B cells in a dose-dependent manner, whereas it had no effect on the protein expression in $\mathrm{HuH7}$ cells (Figure 4b). RT-PCR confirmed this effect; mRNA levels were upregulated by the demethylator in Hep3B cells, but not in HuH7 cells. In other words, the demethylating agent dose-dependently reactivated the Cox-2 transcription that had been silenced by promoter hypermethylation.

To examine the phenotypic change by COX-2 induction, cyclin D1, a hallmark of cell turnover, was examined by Western blotting (Figure 4). The 


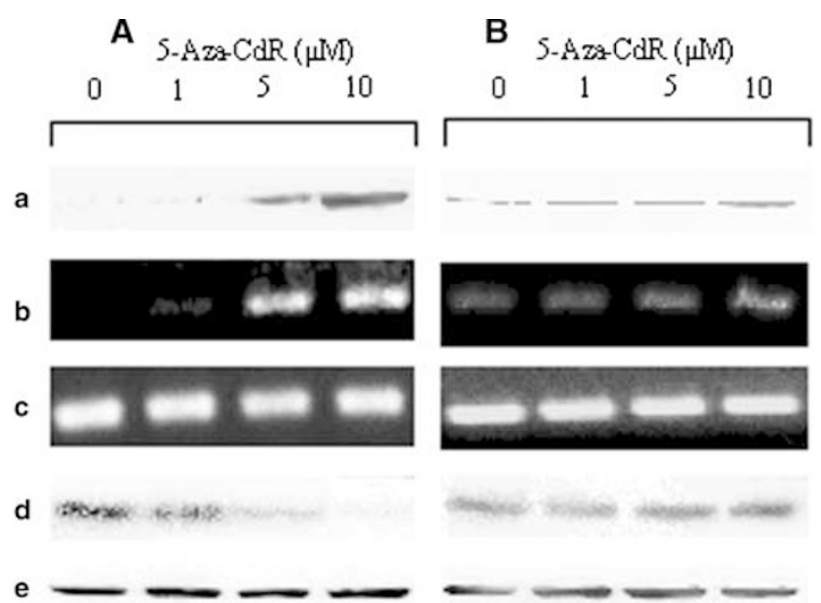

Figure 4 COX-2 restoration and cyclin D1 down-regulation by 5-Aza-CdR. (A) Hep3B cells, and (B) HuH7 cells. Treatment with 5-Aza-CdR induced COX-2 protein synthesis (a) (Western blotting) as well as transcriptional activation of the Cox-2 gene (b) (RT-PCR) dose-dependently in Hep3B cells, but not in HuH7 cells. To confirm that the equal amount of the template was used for RTPCR, the GAPDH gene was amplified (c). The Western blot used for COX-2 (a) was reprobed with cyclin D1 antibody. Cyclin D1 was downregulated along with the COX-2 restoration in Hep3B cells. No such change was observed in $\mathrm{HuH7}$ cells (d). Western blotting for GAPDH confirmed that the equal amount of protein was loaded (e). The experiments were repeated three times and provided similar results.

blot used to examine COX-2 expression was reprobed with cyclin D1 antibody. Blots for GAPDH confirmed that the equal amount of protein was loaded. Of interest, 5-Aza-CdR treatment, in association with COX-2 induction, inhibited the protein synthesis of cyclin D1 in a dose-dependent manner, which implicated decelerated cell proliferation by demethylation. Therefore, the possibility was suggested that COX-2 restoration might delay cell cycle and COX-2 inhibition, in turn, might stimulate the growth of HCC cells.

\section{5-Aza-CdR Treatment Increased $\mathrm{PGE}_{2}$ Production via Enhanced COX-2 Activities}

Figure $5 \mathrm{a}$ and $\mathrm{b}$ demonstrate $\mathrm{PGE}_{2}$ synthesis in Hep3B and HuH7 cells after 5-Aza-CdR treatment. In parallel with the enhanced COX-2 expression, $\mathrm{PGE}_{2}$ synthesis was stimulated by 5 -Aza-CdR in a dosedependent manner in Hep3B cells, and $\mathrm{PGE}_{2}$ levels were significantly higher by the treatment at 5 and $10 \mu \mathrm{M}$ than in the control $(P<0.05$ and $P<0.01$, respectively). On the other hand, 5-Aza-CdR had no effect on the synthesis of $\mathrm{PGE}_{2}$ and $\mathrm{PGE}_{2}$ levels remained relatively low in $\mathrm{HuH7}$ cells.

To confirm that the increased $\mathrm{PGE}_{2}$ production by 5-Aza-CdR treatment in Hep3B cells was attributable to the enhanced COX-2 protein expression, the cells were treated with a COX-2-specific inhibitor celecoxib in combination with 5-Aza-CdR, and $\mathrm{PGE}_{2}$ production was measured (Figure 5c).

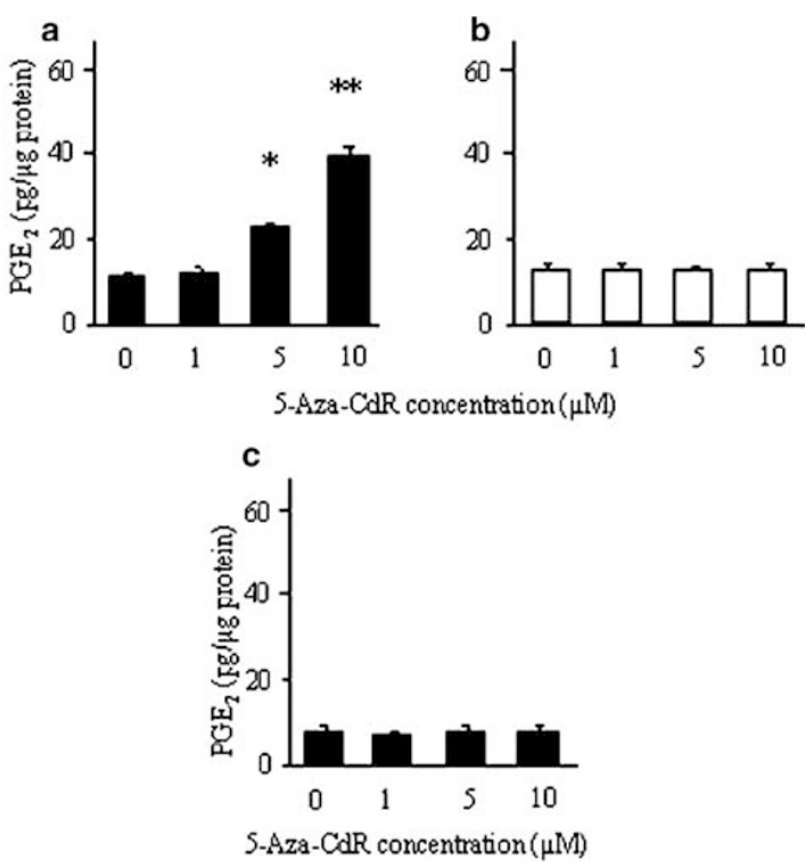

Figure $5 \mathrm{PGE}_{2}$ production by 5 -Aza-CdR and celecoxib. Treatment with 5-Aza-CdR for $96 \mathrm{~h}$ significantly stimulated $\mathrm{PGE}_{2}$ production dose dependently in Hep3B cells (a), while no change was observed in HuH7 cells (b). The increased $\mathrm{PGE}_{2}$ production in Hep3B cells was abrogated by $1 \mu \mathrm{M}$ of celecoxib treatment (c). * and ${ }^{*}$ denote $P<0.05$ and $P<0.01$ vs the control, respectively. $\mathrm{PGE}_{2}$ production was measured in quadruplicate in three independent experiments.

In the controls (without 5-Aza-CdR treatment), $\mathrm{PGE}_{2}$ production in Hep3B cells was comparable between with and without celecoxib treatment (Figure 5a and c), indicating that COX-2 inhibition had little effect on $\mathrm{PGE}_{2}$ production in the controls. This result suggested that a majority of $\mathrm{PGE}_{2}$ production was catalyzed by COX-1 instead of COX-2 in Hep3B cells under the regular culture condition. In Hep3B cells treated with celecoxib, $\mathrm{PGE}_{2}$ levels were similar and unaffected by 5-AzaCdR (Figure 5c). Collectively, this implied that the restored COX-2 activity was essentially abrogated by $1 \mu \mathrm{M}$ of celecoxib. Conversely, as shown in Figure $5 \mathrm{a}$, an increase in $\mathrm{PGE}_{2}$ production by 5 -Aza-CdR treatment was mainly attributable to COX-2 induction.

\section{5-Aza-CdR Treatment Suppressed Cell Growth}

To explore the outcome of the COX-2 restoration by 5-Aza-CdR, changes in numbers of Hep3B cells were examined by WST-8 assay. As shown in Figure 6a (closed bars), $96 \mathrm{~h}$ of treatment with 5-Aza-CdR significantly inhibited the growth of Hep3B cells in a dose-dependent manner $(P<0.01$ vs the control).

Next, to determine whether the enhanced COX-2 expression by 5-Aza-CdR inhibited cell growth, Hep3B cells were treated with celecoxib to suppress COX-2 activity and the cell growth was examined. 
Celecoxib, which suppressed COX-2 activity and $\mathrm{PGE}_{2}$ production almost completely at $1 \mu \mathrm{M}$ in the same cells (Figure 5c), reversed the growth inhibitory effect of 5-Aza-CdR (Figure 6a, open bars). In the presence of celecoxib, there was no significant change in the cell number at any concentrations of 5-Aza-CdR, compared with the control without 5-Aza-CdR treatment $(0 \mu \mathrm{M})$. This result implicated that COX-2 reactivation indeed suppressed the growth of Hep3B cells. Interestingly, without the addition of 5-Aza-CdR (at $0 \mu \mathrm{M}$ ), celecoxib suppressed the growth of Hep3B cells. As shown in Figure 5a and c, celecoxib hardly affected the production of $\mathrm{PGE}_{2}$ in the absence of 5 -Aza-CdR $(0 \mu \mathrm{M})$, indicating that this growth suppression was mediated by the specific effect of celecoxib, rather than COX-2 inhibition or suppression of $\mathrm{PGE}_{2}$ production.

The growth inhibition of Hep3B cells by the demethylator treatment was predicted in view of the previous result on cyclin D1. In agreement with cyclin D1 downregulation (Figure 4), restored COX2 activities might delay cell growth, and the possibility was indicated that COX-2 suppression might accelerate the development of HCC.

On the other hand, 5-Aza-CdR treatment up to $10 \mu \mathrm{M}$ had little effect on the growth of HuH7 cells (Figure 6b, closed bars), which was in good contrast with the growth inhibition observed in Hep3B cells (Figure 6a). This result was expected because neither COX-2 expression nor $\mathrm{PGE}_{2}$ production was unaltered by the demethylator in $\mathrm{HuH} 7$ cells (Figures 4b and 5b). As was found in Hep3B cells, celecoxib suppressed the growth of $\mathrm{HuH7}$ cells in the absence of 5-Aza-CdR $(0 \mu \mathrm{M})$ (Figure 6b, open bars). Moreover, celecoxib, to a similar extent, suppressed the growth of $\mathrm{HuH7}$ cells at all the examined concentrations of 5-Aza-CdR (Figure 6b, open bars). Considering that $\mathrm{PGE}_{2}$ was synthesized at a low level and 5-Aza-CdR hardly affected COX-2 expression or $\mathrm{PGE}_{2}$ production in $\mathrm{HuH7}$ cells (Figures $4 \mathrm{~b}$ and $5 \mathrm{~b}$ ), the result that the growth of $\mathrm{HuH7}$ cells was inhibited also implicated the specific growth-inhibitory effect of celecoxib, rather than the outcome of COX-2 inhibition.

Taken together, despite the specific growth-inhibitory effect of celecoxib, at least found in these two cell lines, COX-2 reactivation by the demethylation of the Cox-2 promoter delayed the growth of Hep3B cells.

\section{Cell Viability by 5-Aza-CdR}

To exclude the possibility that the growth of Hep3B cells was suppressed due to the cytotoxicity of 5 -Aza-CdR, LDH release into the supernatant was measured. Up to $10 \mu \mathrm{M}$ of 5 -Aza-CdR treatment, there was no increase in LDH values in either Hep3B or $\mathrm{HuH7}$ cells, indicating that these cells were hardly killed by 5-Aza-CdR at the concentrations
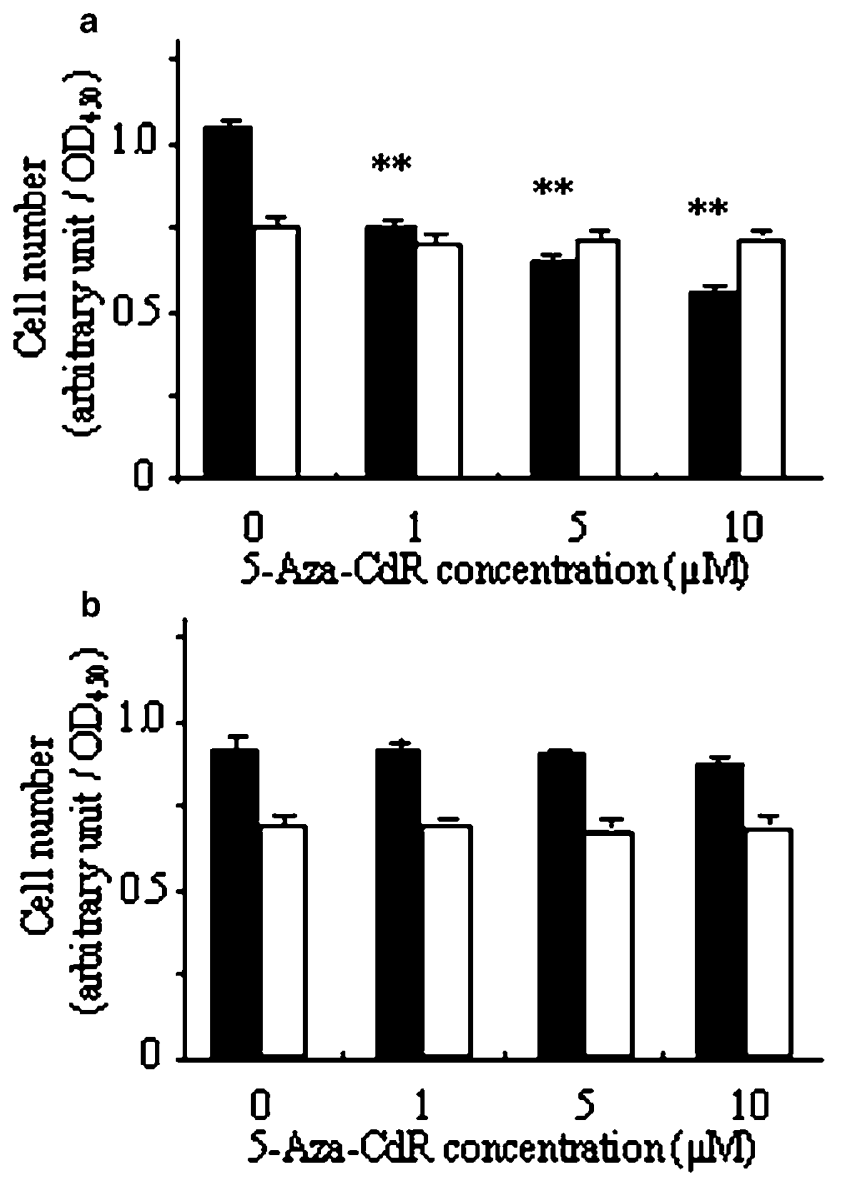

Figure 6 Cell growth by 5-Aza-CdR and celecoxib. Cell viability was determined by the WST-8 assay. (a) 5-Aza-CdR inhibited growth of Hep3B cells dose dependently (closed bars), and the growth inhibition was abolished to some extent by $1 \mu \mathrm{M}$ of celecoxib treatment (open bars). (b) Dose-dependent growth inhibition by 5 -Aza-CdR was not found in HuH7 cells (closed bars), and celecoxib treatment showed similar growth suppression irrespective of 5-Aza-CdR concentrations (open bars), indicating the specific growth-inhibitory effect of celecoxib. ** denotes $P<0.01$ vs the control. The WST-8 assay was carried out in quadruplicate in three independent experiments.

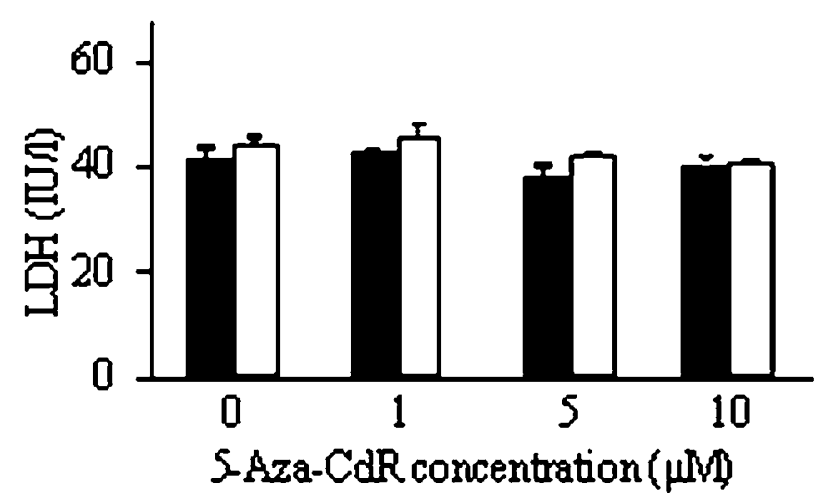

Figure 7 No remarkable cytotoxicity by 5-Aza-CdR. Cytotoxicity and cell viability by 5 -Aza-CdR treatment were examined by the LDH release into the supernatant. No remarkable cytotoxicity or impaired viability was observed in Hep3B (closed bars) and HuH7 cells (open bars). 
examined (Figure 7). This result also supports the idea that the growth of Hep3B cells was inhibited not by the cytotoxicity of 5 -Aza-CdR but by COX-2 reactivation.

\section{Discussion}

A number of reports have demonstrated that COX-2 upregulation is involved in carcinogenesis. ${ }^{14-29}$ Among them, it is particularly intriguing that COX2 expression is highly upregulated in early HCC, but relatively downregulated over progression. ${ }^{23-29}$ However, the underlying mechanism to regulate COX-2 expression, especially in HCC, is poorly understood. This prompted us to hypothesize that COX-2 expression could be regulated by promoter hypermethylation in HCC cells.

In the present study, we provided compelling evidence that $\mathrm{CpG}$ sites in the Cox-2 promoter in some HCC cell lines were hypermethylated and 5-Aza-CdR treatment could demethylate the promoter in Hep3B cells that originally had hypermethylated Cox-2 promoter, resulting in the transcriptional activation of the Cox-2 gene. In addition, we demonstrated that COX-2 reactivation by treatment with 5-Aza-CdR suppressed the growth of Hep3B cells.

In an attempt to clarify the methylation status of the Cox-2 promoter, three $\mathrm{CpG}$ sites were examined: $-431 \mathrm{nt}(\mathrm{A}),-372 \mathrm{nt}(\mathrm{B})$, and $-138 \mathrm{nt}$ (C) from the transcription starting site. It is generally accepted that COX-2 expression is regulated predominantly by the activation of three transcription factors: NF$\kappa \mathrm{B}$, NF-IL6, and CRE. The consensus sequences for NF- $\kappa \mathrm{B}(-452 /-433,-229 /-209)$, NF-IL6 $(-132 /$ $-124)$, and CRE $(-59 /-53)$ binding sites are known (Figure 1, GenBank: AF276953). ${ }^{4,53-55}$ The CpG sites (A) and (B) were located not within but close to the NF- $\kappa$ B site, while the CpG site (C) was also not within but near the NF-IL6 site. Both NF- $\kappa$ B and NFIL6 sites were greatly involved in the transcription of Cox-2. ${ }^{55}$ Hence, there is a substantial possibility that the methylation of $\mathrm{CpG}$ sites at these sites repressed Cox-2 transcription in Hep3B cells under the regular culture condition, and demethylation by 5-Aza-CdR at these sites, probably through creating newly accessible NF- $\kappa \mathrm{B}$ and NF-IL6 binding sites for each transcription factor, reactivated Cox-2 transcription.

DNA methylation has been shown to reduce the binding affinity of sequence-specific transcription factors. ${ }^{56,57}$ Furthermore, methylation-dependent, sequence-specific DNA-binding proteins, such as MDBP, may act as transcriptional repressors. ${ }^{58}$ However, it has also been reported that the transcriptional repression by DNA methylation can be mediated through a sequence-independent process involving changes in chromatin structure and histone acetylation levels. ${ }^{59,60}$ In general, CpG sites in the promoter are not uniformly methylated, ${ }^{44,61}$ and there is a mixed population of methylated and unmethylated clones at any $\mathrm{CpG}$ site. Indeed, as shown in Figure 2, heterogeneity was observed in the methylation status of $\mathrm{CpG}$ sites. As to the Cox-2 gene, there are $51 \mathrm{CpG}$ sites in the promoter region from -590 to $+186 \mathrm{nt}^{44}$ Collectively, it needs further investigation to conclude which $\mathrm{CpG}$ site is pivotal in regulating Cox-2 transcription and thereby causing a change in chromatin structure and accessibility of transcription factors to the binding sites. However, we could at least demonstrate that the methylation status of the $\mathrm{CpG}$ sites examined in this study was associated with the COX-2 expression and these sites seemed to play potentially a critical role in the transcriptional repression and reactivation.

Recently a high level of cancer-specific methylation in human cancer has been referred to as the CpG island methylator phenotype (CIMP). ${ }^{61}$ Tumors exhibiting this phenotype are characterized by a high degree of concurrent $\mathrm{CpG}$ island methylation in a variety of genes, such as $p 16, h M L H 1$, and THBS1. In HCC cells with hypermethylated Cox-2 promoter including Hep3B cells, promoters of other genes can also be hypermethylated; in fact, promoters in the estrogen receptor, $p 16$, and T-type calcium channel gene are hypermethylated. ${ }^{46,62}$

In Hep3B cells, promoter hypermethylation was found in the Ras association domain family $1 \mathrm{~A}$ (RASSF1A) gene, ${ }^{63}$ pi-class glutathione $S$-transferase (GSTP1) gene, ${ }^{64-66}$ but not in $p 16 .{ }^{63}$ Interestingly, promoter hypermethylation of RASSF1A was found in $85 \%$ of HCC tissues examined while in $0 \%$ in the adjacent nontumorous liver tissues. ${ }^{63,67}$ These previous reports indicate that the methylation status in human HCC tissue may be, to some extent, retained in some HCC cell lines as well.

Treatment with 5-Aza-CdR may demethylate and reactivate other genes involved in cell growth, such as the $p 16$ gene, and result in growth suppression. Therefore, the involvement of such genes cannot be completely excluded in interpreting the results of the experiments using a demethylator. However, considering that 5-Aza-CdR, in the presence of celecoxib, did not affect the growth of HuH7 cells in a dose-dependent manner, the abrogation of cell growth suppression by celecoxib found in Hep3B cells implicated that reactivated COX-2 by promoter demethylation, rather than reactivation of other formerly methylated genes, predominantly suppressed or delayed the cell growth.

Intestinal epithelial cells overexpressing COX-2 have the increased duration of the $G_{1}$ phase of cell cycle, lower levels of cyclin D1 protein, and a marked decrease in retinoblastoma kinase activity associated with cyclin-dependent kinase $4 .^{30}$ In addition, overexpression of COX-2 arrested cell cycle in other cell types such as fibroblasts and endothelial cells. ${ }^{31}$ Furthermore, there have been a number of reports that $\mathrm{PGE}_{2}$ and its derivatives suppressed growth of a variety of cells, ${ }^{32-37}$ whereas 
they could also stimulate colony formation and transactivate EGF receptor, which presumably led to cell proliferation. ${ }^{38,39}$

Not surprisingly, consistent with these reports showing that upregulated COX-2 and $\mathrm{PGE}_{2}$ delayed cell growth, our results provided evidence that growth of HCC cells was suppressed or retarded, at least in part, by demethylation of the Cox-2 promoter, that is, COX-2 reactivation, and it was related with cyclin D1 downregulation. We could also demonstrate that treatment with a COX-2 inhibitor abrogated the cell growth suppression by 5 -Aza-CdR. Therefore, in spite of the possible involvement of other genes whose promoters are originally methylated and can be demethylated by a demethylator, COX-2 reactivation seems to play a major causative role in growth inhibition in HCC. In this study, the effect of PGs on cell cycle was not directly investigated in HCC cells. We are currently investigating the effect of PGs, especially $\mathrm{PGE}_{2}$, on cell cycle in HCC cells.

The underlying mechanism how the Cox-2 promoter becomes hypermethylated is poorly understood. DNA methyltransferases are presumed to play a significant role in DNA methylation. Compared with the corresponding noncancerous liver tissue, significant overexpression of DNMT3b, which had de novo DNA methylation activity, ${ }^{68}$ was observed in HCC. ${ }^{62}$ Nevertheless, DNMT3b mRNA levels did not change significantly over dedifferentiation, ${ }^{62}$ and whether this mechanism may be involved in the promoter hypermethylation in HCC cells remains unclear.

Methyl-CpG binding domain (MBD) proteins, at least in part, mediate CpG island hypermethylation and repress gene transcription. Bakker et $a l^{64}$ demonstrated that MBD2, among MBD family proteins, repressed GSTP1 transcription via hypermethylation of the promoter in Hep3B cells. Therefore, although the exact mechanism how the Cox-2 promoter can be hypermethylated remains unclear and needs further investigation, MBD2 is a potential candidate that mediated the hypermethylation process.

Given that the $\mathrm{IC}_{50}$ of celecoxib is about $0.04 \mu \mathrm{M}$, the concentration of celecoxib used in this study $(1 \mu \mathrm{M})$ was considered reasonably enough to suppress the COX-2 activity. Indeed, judging from the reduced $\mathrm{PGE}_{2}$ production, COX-2 inhibitor celecoxib almost completely abolished the COX-2 activity restored by 5-Aza-CdR. In contrast, COX-2-specific inhibitors are known to have proapoptotic and antiproliferative potentials in vitro at markedly high concentrations $(\sim 100 \mu \mathrm{M}) .{ }^{26,69}$ Should COX-2 inhibitors be used at such high concentrations, they may exhibit chemopreventive effect on HCC. This possibility must be investigated in vivo in the future.

In conclusion, our present study indicates that promoter hypermethylation silences the Cox-2 gene and the reactivation of $\mathrm{Cox}-2$ may lead to growth retardation in some HCC cell lines. Although we did not investigate on human HCC tissue in this study, it would be intriguing to examine the distribution patterns of the cells with hypermethylated Cox-2 promoter within tumors.

\section{Acknowledgement}

This study was in part supported by the Ministry of Education, Culture, Sports, Science and Technology, Japan.

\section{References}

1 Jones PA, Laird PW. Cancer epigenetics comes of age. Nature Genet 1999;21:163-167.

2 Baylin SB, Herman JG, Graff JR, et al. Alterations in DNA methylation: a fundamental aspect of neoplasia. Adv Cancer Res 1998;72:141-146.

3 Jones PA, Baylin SB. The fundamental role of epigenetic events in cancer. Nat Rev Genet 2002;3:415-428.

4 Wutz A, Smrzka OW, Schweifer N, et al. Imprinted expression of the Igf2r gene depends on an intronic CpG island. Nature 1997;389:745-749.

5 Stögert RP, Kubicka P, Liu CG, et al. Maternal-specific methylation of the imprinted mouse Igf2r locus identifies the expressed locus as carrying the imprinting signal. Cell 1993;73:61-71.

6 Counts JL, Goodman JI. Alterations in DNA methylation may play a variety of roles in carcinogenesis. Cell 1995;83:13-15.

7 Warnecke PM, Bestor TH. Cytosine methylation and human cancer. Curr Opin Oncol 2000;12:68-73.

8 Bird A. DNA methylation. Science (Wash DC) 1999; 286:2287-2288.

9 Jones PA. DNA methylation errors and cancer. Cancer Res 1996;56:2463-2467.

10 Kujubu DA, Fletcher BS, Varnum BC, et al. TIS10, a phorbol ester tumor promoter-inducible mRNA from Swiss 3T3 cells, encodes a novel prostaglandin synthase/cyclooxygenase homologue. J Biol Chem 1991;266:12866-12872.

11 Maier JA, Hla T, Maciag T. Cyclooxygenase is an immediate-early gene induced by interleukin-1 in human endothelial cells. J Biol Chem 1990;265: 10805-10808.

12 Inoue H, Yokoyama C, Hara S, et al. Transcriptional regulation of human prostaglandin-endoperoxide synthase-2 gene by lipopolysaccharide and phorbol ester in vascular endothelial cells. J Biol Chem 1995;270:24965-24971.

13 Ristimäki A, Garfinkel S, Wessendorf J, et al. Induction of cyclooxygenase-2 by interleukin-1 alpha. Evidence for post-transcriptional regulation. J Biol Chem 1994;269:11769-11775.

14 Eberhart CE, Coffey RJ, Radhika A, et al. Up-regulation of cyclooxygenase 2 gene expression in human colorectal adenomas and adenocarcinomas. Gastroenterology 1994;197:1183-1188.

15 Murata H, Kawano S, Tsuji S, et al. Cyclooxygenase-2 overexpression enhances lymphatic invasion and metastasis in human gastric carcinoma. Am J Gastroenterol 1999;94:451-455. 
16 Wilson KT, Fu S, Ramanujam KS, et al. Increased expression of inducible nitric oxide synthase and cyclooxygenase-2 in Barrett's esophagus and associated adenocarcinoma. Cancer Res 1998;58: 2929-2934.

17 Tucker ON, Dannenberg AJ, Yang EK, et al. Cyclooxygenase-2 expression is up-regulated in human pancreatic cancer. Cancer Res 1999;59:198-204.

18 Wolff H, Saukkonen K, Anttila S, et al. Expression of cyclooxygenase-2 in human lung carcinoma. Cancer Res 1998;58:4997-5001.

19 Tsujii M, DuBois RN. Alterations in cellular adhesion and apoptosis in epithelial cells overexpressing prostaglandin endoperoxide synthase-2. Cell 1995;83: 493-501.

20 Tsujii M, Kawano S, Tsuji S, et al. Cyclooxygenase regulates angiogenesis induced by colon cancer cells. Cell 1998;93:705-716.

21 Tsujii M, Kawano S, DuBois RN. cyclooxygenase-2 expression in human colon cancer cells increases metastatic potential. Proc Natl Acad Sci USA 1997; 94:3336-3340.

22 Kakiuchi Y, Tsuji S, Tsujii M, et al. Cyclooxygenase-2 activity altered the cell-surface carbohydrate antigens on colon cancer cells and enhanced liver metastasis. Cancer Res 2002;62:1567-1572.

23 Fantappiè O, Masini E, Sardi I, et al. The MDR phenotype is associated with the expression of COX2 and iNOS in a human hepatocellular carcinoma cell line. Hepatology 2002;35:843-852.

24 Koga H, Sakisaka S, Ohishi M, et al. Expression of cyclooxygenase-2 in human hepatocellular carcinoma: relevance to tumor dedifferentiation. Hepatology 1999; 29:688-696.

25 Rahman MA, Dhar DK, Yamaguchi E, et al. Coexpression of inducible nitric oxide synthase and COX-2 in hepatocellular carcinoma and surrounding liver: possible involvement of COX-2 in the angiogenesis of hepatitis C virus-positive cases. Clin Cancer Res 2001;7:1325-1332.

26 Bae SH, Jung ES, Park YM, et al. Expression of cyclooxygenase-2 (COX-2) in hepatocellular carcinoma and growth inhibition of hepatoma cell lines by a COX-2 inhibitor, NS-398. Clin Cancer Res 2001;7: 1410-1418.

27 Shiota G, Okubo M, Noumi T, et al. Cyclooxygenase-2 expression in hepatocellular carcinoma. Hepatogastroenterol 1999;46:407-412.

28 Kondo M, Yamamoto $\mathrm{H}$, Nagano $\mathrm{H}$, et al. Increased expression of COX-2 in nontumor liver tissue is associated with shorter disease-free survival in patients with hepatocellular carcinoma. Clin Cancer Res 1999;5:4005-4012.

29 Cheng J, Imanishi $\mathrm{H}$, Iijima $\mathrm{H}$, et al. Expression of cyclooxygenase 2 and cytosoloic phospholipase A2 in the liver tissue of patients with chronic hepatitis and liver cirrhosis. Hepatol Res 2002;23:185-195.

30 DuBois RN, Shao J, Tsujii M, et al. G1 delay in cells overexpressing prostaglandin endoperoxide synthase2. Cancer Res 1996;56:733-737.

31 Trifan OC, Smith RM, Thompson BD, et al. Overexpression of cyclooxygenase-2 induces cell cycle arrest. J Biol Chem 1999;274:34141-34147.

32 Santoro MG, Philpott GW, Jaffe BM. Inhibition of tumour growth in vivo and in vitro by prostaglandin $\mathrm{E}$. Nature 1976;263:777-779.
33 Prasad KN. Morphological differentiation induced by prostaglandin in mouse neuroblastoma cells in culture. Nat New Biol 1972;236:49-52.

34 Shimakura S, Boland CR. Eicosanoid production by the human gastric cancer cell line AGS and its relation to cell growth. Cancer Res 1992;52:1744-1749.

35 Nakamura A, Yamatani T, Fujita T, et al. Mechanism of inhibitory action of prostaglandins on the growth of human gastric carcinoma cell line KATO III. Gastroenterology 1991;101:910-918.

36 Derubertis FR, Craven PA, Saito R. 16, 16-dimethyl prostaglandin E2 suppresses the increases in the proliferative activity of rat colonic epithelium induced by indomethacin and aspirin. Gastroenterology 1985;89:1054-1063

37 Craven PA, Saito R, Derubertis FR. Role of local prostaglandin synthesis in the modulation of proliferative activity of rat colonic epithelium. J Clin Invest 1983;72:1365-1375.

38 Sheng H, Shao J, Washingon MK, et al. Prostaglandin E2 increases growth and motility of colorectal carcinoma cells. J Biol Chem 2001;276:18075-18081.

39 Pai R, Soreghan B, Szabo IL, et al. Prostaglandin E2 transactivates EGF receptor: a novel mechanism for promoting colon cancer growth and gastrointestinal hypertrophy. Nat Med 2002;8:289-293.

40 Thun MJ, Namboodiri MM, Heath CW. Aspirin use and reduced risk of fatal colon cancer. New Engl J Med 1991;325:1593-1596.

41 Giovannucci E, Egan KM, Hunter DJ, et al. Aspirin and the risk of colorectal cancer in women. New Engl J Med 1995;333:609-614.

42 Thun MJ, Namboodiri MM, Calle EE, et al. Aspirin use and risk of fatal cancer. Cancer Res 1993;53:1322-1327.

43 Toyota M, Shen L, Ohe-Toyota M, et al. Aberrant methylation of the cyclooxygenase $2 \mathrm{CpG}$ island in colorectal tumors. Cancer Res 2000;60:4044-4048.

44 Song SH, Jong HS, Choi HH, et al. Transcriptional silencing of cyclooxygenase-2 by hyper-methylation of the $5^{\prime} \mathrm{CpG}$ island in human gastric carcinoma cells. Cancer Res 2001;61:4628-4635.

45 Akhtar M, Cheng Y, Romina M, et al. Promoter methylation regulates Helicobacter pylori-stimulated cyclooxygenase-2 expression in gastric epithelial cells. Cancer Res 2001;61:2399-2403.

46 Shen L, Ahuja N, Shen Y, et al. DNA methylation and environmental exposures in human hepatocellular carcinoma. J Natl Cancer Inst 2002;94:755-761.

47 Aden DP, Fogel A, Plotkin S, et al. Controlled synthesis of HBsAg in a differentiated human liver carcinomaderived cell line. Nature 1979;282:615-616.

48 Hsu IC, Tokiwa T, Bennett W, et al. p53 gene mutation and integrated hepatitis B viral DNA sequences in human liver cancer cell lines. Carcinogenesis 1993; 4:987-992.

49 Kane MF, Loda M, Gaida GM, et al. Methylation of the hMLH1 promoter correlates with lack of expression of hMLH1 in sporadic colon tumors and mismatch repair-defective human tumor cell lines. Cancer Res 1997;57:808-811.

50 Takahashi M, Katayama Y, Takada $\mathrm{H}$, et al. The effect of NSAIDs and a COX-2 specific inhibitor on Helicobacter pylori-induced PGE2 and HGF in human gastric fibroblasts. Aliment Pharmacol Ther 2000;14 (Suppl 1):44-49.

51 Bird AP. CpG-rich islands and the function of DNA methylation. Nature (London) 1986;321:209-213. 
52 Gardiner-Garden M, Frommer M. CpG islands in vertebrate genomes. J Mol Biol 1987;196:261-282.

53 Lukiw WJ, Pelaez RP, Martinez J, et al. Budenoside epimer $\mathrm{R}$ or dexamethasone selectively inhibit platelet-activating factor-induced or interleukin 1beta-induced DNA binding activity of cis-acting transcription factors and cyclooxygenase-2 gene expression in human epidermal keratinocytes. Proc Natl Acad Sci USA 1998;95:3914-3919.

54 Inoue H, Nanayama T, Hara S, et al. The cyclic AMP response element plays an essential role in the expression of the human prostaglandin-endoperoxide synthase 2 gene in differentiated U937 monocytic cells. FEBS Lett 1994;350:51-54.

55 Yamamoto K, Arakawa T, Ueda N, et al. Transcriptional role of nuclear factor kappaB and nuclear factor-interluekin-6 in the tumor necrosis factor alphadependent induction of cyclooxygenase-2 in MC3T3E1 cells. J Biol Chem 1995;270:31315-31320.

56 Clark SJ, Harrison J, Molloy PL. Sp1 binding is inhibited by $(\mathrm{m}) \mathrm{Cp}(\mathrm{m}) \mathrm{CpG}$ methylation. Gene 1997;195:67-71.

57 Prendergast GC, Ziff EB. Methylation-sensitive sequence-specific DNA binding by the c-Myc basic region. Science 1991;251:186-189.

58 Asiedu CK, Scotto L, Assoian RK, et al. Binding of AP1/CREB proteins and of MDBP to contiguous sites downstream of the human TGF-beta 1gene. Biochim Biophys Acta 1994;1219:55-63.

59 Kass SU, Pruss D, Wolff AP. How does DNA methylation repress transcription? Trends Genet 1997;13:444-449.

60 Bestor TH. Gene silencing. Methylation meets acetylation. Nature 1998;393:311-312.

61 Toyota M, Ahuja N, Ohe-Toyota M, et al. CpG island methylator phenotype in colorectal cancer. Proc Natl Acad Sci USA 1999;96:8681-8686.
62 Saito Y, Kanai Y, Sakamoto M, et al. Overexpression of a splice variant of DNA methyltransferase $3 \mathrm{~b}$, DNMT3b4, associated with DNA hypomethylation on pericentromeric satellite regions during human hepatocarcinogenesis. Proc Natl Acad Sci USA 2002;99: 10060-10065.

63 Zhang YJ, Ahsan H, Chen Y, et al. High frequency of promoter hypermethylation of RASSF1A and p16 and its relationship to aflatoxin B1-DNA adduct levels in human hepatocellular carcinoma. Mol Carcinog 2002;35:85-92.

64 Bakker J, Lin X, Nelson WG. Methyl-CpG binding domain protein 2 represses transcription from hypermethylated pi-class glutathione S-transferase gene promoters in hepatocellular carcinoma cells. J Biol Chem 2002;277:22573-22580.

65 Zhong S, Tang MW, Yeo W, et al. Silencing of GSTP1 gene by $\mathrm{CpG}$ island DNA hypermethylation in HBVFassociated hepatocellular carcinomas. Clin Cancer Res 2002;8:1087-1092.

66 Tchou JC, Lin X, Freije D, et al. GSTP1 CpG island DNA hypermethylation in hepatocellular carcinomas. Int J Oncol 2000;16:663-676.

67 Schagdarsurengin U, Wilkens L, Steinemann D, et al. Frequent epigenetic inactivation of the RASSF1A gene in hepatocellular carcinoma. Oncogene 2003;22: 1866-1871.

68 Okano M, Bell DW, Haber DA, et al. DNA methyltransferases Dnmt3a and Dnmt3b are essential for de novo methylation and mammalian development. Cell 1999;99:247-257.

69 Kern MA, Schubert D, Sahi D, et al. Proapoptotic and antiproliferative potential of selective cyclooxygenase2 inhibitors in human liver tumor cells. Hepatology 2002;36:885-894. 